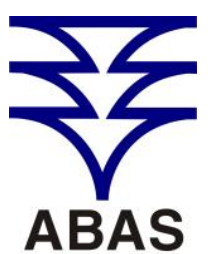

ASSOCIACAO BRASLEERADE
AGUAS SUBTERRANEAS www.abas.org

\section{AVALIAÇÃO ISOTÓPICA E HIDROQUÍMICA DO AQUÍFERO CÁRSTICO SALITRE NA REGIÃO DE IRECÊ, BAHIA}

\author{
ISOTOPIC AND HYDROCHEMISTRY EVALUATION OF SALITRE KARST \\ AQUIFER IN REGION OF IREC $\hat{E}$, BAHIA
}

\begin{abstract}
Tereza Cristina Bittencourt Villanueva ${ }^{1}$, Luiz Rogério Bastos Leal ${ }^{2}$ Maria do Rosario Zucchi ${ }^{3}$, Antonio Expedito Gomes de Azevedo ${ }^{4}$, José Garcia Vivas Miranda ${ }^{5}$, Pablo Ramosandrade Villanueva ${ }^{6}$
\end{abstract}

Artigo recebido em: 29/07/2014 e aceito para publicação em: 08/10/2014.

\begin{abstract}
The research was conducted at Salitre aquifer, located in the region of Irecê in the north-central portion of the state of Bahia. Aims to characterize one hydrochemical and isotopic through the use of stable isotopes of oxygen-18, hydrogen and dissolved inorganic carbon $\left({ }^{18} \mathrm{O}, \delta \mathrm{D}, \delta^{13} \mathrm{C}_{\mathrm{CID}}\right)$ in water samples collected from wells in the Salitre aquifer in the area. The isotopic analyzes obtained in wells located in the eastern sector of the upstream flow have a lower isotopic enrichment than those obtained in wells located in the north-northwest flow downstream sector, demonstrating that the isotopic enrichment occurs especially from East to West, consistent with preferred flow directions obtained in the area, being corroborated by historical isotopic data obtained in Irecê Basin, which reveals that there was no significant isotopic variations Salitre aquifer over the past 30 years.
\end{abstract}

Keywords: Salitre karst aquifer. Stable isotopes. Principal Component Analysis.

Resumo: A pesquisa foi desenvolvida no aquífero Salitre, localizado na região de Irecê, na porção centro-norte do estado da Bahia. Tem como objetivo uma caracterização hidroquímica e isotópica, através da utilização de isótopos estáveis de hidrogênio, oxigênio e carbono inorgânico dissolvido $\left(\delta \mathrm{D}, \delta^{18} \mathrm{O}, \delta^{13} \mathrm{C}_{\mathrm{CID}}\right)$ em amostras de água coletadas em poços tubulares do aquífero Salitre na área. As análises isotópicas obtidas em poços tubulares localizados no setor leste à montante do fluxo apresentam um menor enriquecimento isotópico do que aquelas obtidas em poços localizados no setor norte-noroeste à jusante do fluxo, demonstrando que o enriquecimento isotópico ocorre, sobretudo, de Leste para Oeste, concordante com as direções preferenciais de fluxo obtidas na área, sendo corroborado pelos dados isotópicos históricos obtidos na Bacia de Irecê, que revela que não houve uma variação isotópica significativa do aquífero Salitre ao longo dos últimos 30 anos.

Palavras-chave: Aquífero cárstico Salitre. Isótopos estáveis. Análise de Componentes Principais.

\title{
INTRODUÇÃO
}

O monitoramento das águas subterrâneas, de acordo com o manual da UNESCO (Vrba e Soblsek, 1988), é um dos instrumentos mais importantes para a viabilização de estratégias e políticas de proteção e conservação desses recursos. A implantação de programas de monitoramento das águas subterrâneas auxilia na melhoria do seu planejamento, proteção e manejo. Dessa forma, de acordo com Andreo et al., (2003), a análise de isótopos é um dos métodos mais adequados para contribuir com a preservação da qualidade dos recursos hídricos subterrâneos.
A compreensão sobre o comportamento da zona insaturada em aquíferos cársticos é de grande importância, de acordo com Carrasco et al., (2006), uma vez que envolve o entendimento sobre os processos de carstificação, bem como os tipos de fluxos hídricos que podem ocorrer no sistema.

Diferentes métodos podem ser utilizados para analisar a zona insaturada em aquíferos cársticos (Bakalowicz, 1995), um dos quais é a análise da composição isotópica das águas de infiltração. Os isótopos estáveis, de acordo com Blavoux et

\footnotetext{
1Pesquisadora em Geociências do Serviço Geológico do Brasil - CPRM (tereza.villanueva@cprm.gov.br). 2Professor Associado do Instituto de Geociências da Universidade Federal da Bahia (lrogerio@ufba.br). 3Professora do Instituto de Física da Universidade Federal da Bahia (maria.zucchi@ gmail.com).

4Professor do Instituto de Física da Universidade Federal da Bahia (expedito@ufba.br).

${ }^{5}$ Professor do Instituto de Física da Universidade Federal da Bahia (vivasm@gmail.com).

${ }^{6}$ Analista Ambiental do Ministério do Meio Ambiente (pablo.villanueva@mma.gov.br).
} 
al., (1979), Mudry (1981), Cruz-San Julián et al., (1992), Lastennet (1994) e Vallejos (1997), são traçadores naturais largamente aplicados na hidrogeologia cárstica.

Os aquíferos cársticos são particularmente vulneráveis à contaminação, como constatado por Goldscheider (2002), devido a fatores como: baixa espessura dos solos, concentração de fraturamentos e carstificação das camadas superiores do aquífero, além da ocorrência de pontos de recarga em cavidades e condutos formados pelo processo de carstificação, que propiciam a penetração e o transporte de contaminantes nesse sistema.

Na região de Irecê, localizada na porção centro-norte do estado da Bahia, o controle estrutural representado por lineamentos e fraturamentos é o principal responsável pelo modelamento das formas típicas de carstificação, que resultam da ação da água sobre a rocha calcária, solubilizando-a e transportando seus produtos. A intensidade do processo depende tanto de fatores geológicos estruturais, como também da quantidade e qualidade química da água, composição da rocha e dos fatores climáticos, sobretudo dos índices de precipitação (NOSSA, 2011).

$\mathrm{Na}$ área, as águas subterrâneas são intensamente utilizadas para a irrigação, devido à pequena disponibilidade de água superficial. Apesar de ser uma região de grande importância agrícola, é pouco estudada do ponto de vista da dinâmica hidrogeológica, o que motivou a escolha do tema na área em questão, além de ser caracterizada pela ocorrência de rochas calcárias, com estruturas cársticas condicionadas por sistemas de fraturamentos, que propicia ao aquífero um elevado potencial de vulnerabilidade intrínseca.

\section{REVISÃO DA LITERATURA}

\section{Características da área de estudo}

A área de pesquisa é formada por um polígono que compreende parte dos municípios de Irecê e Lapão, no estado da Bahia, com uma área de aproximadamente $250 \mathrm{~km}^{2}$ (Figura 1); (NOSSA, 2011).

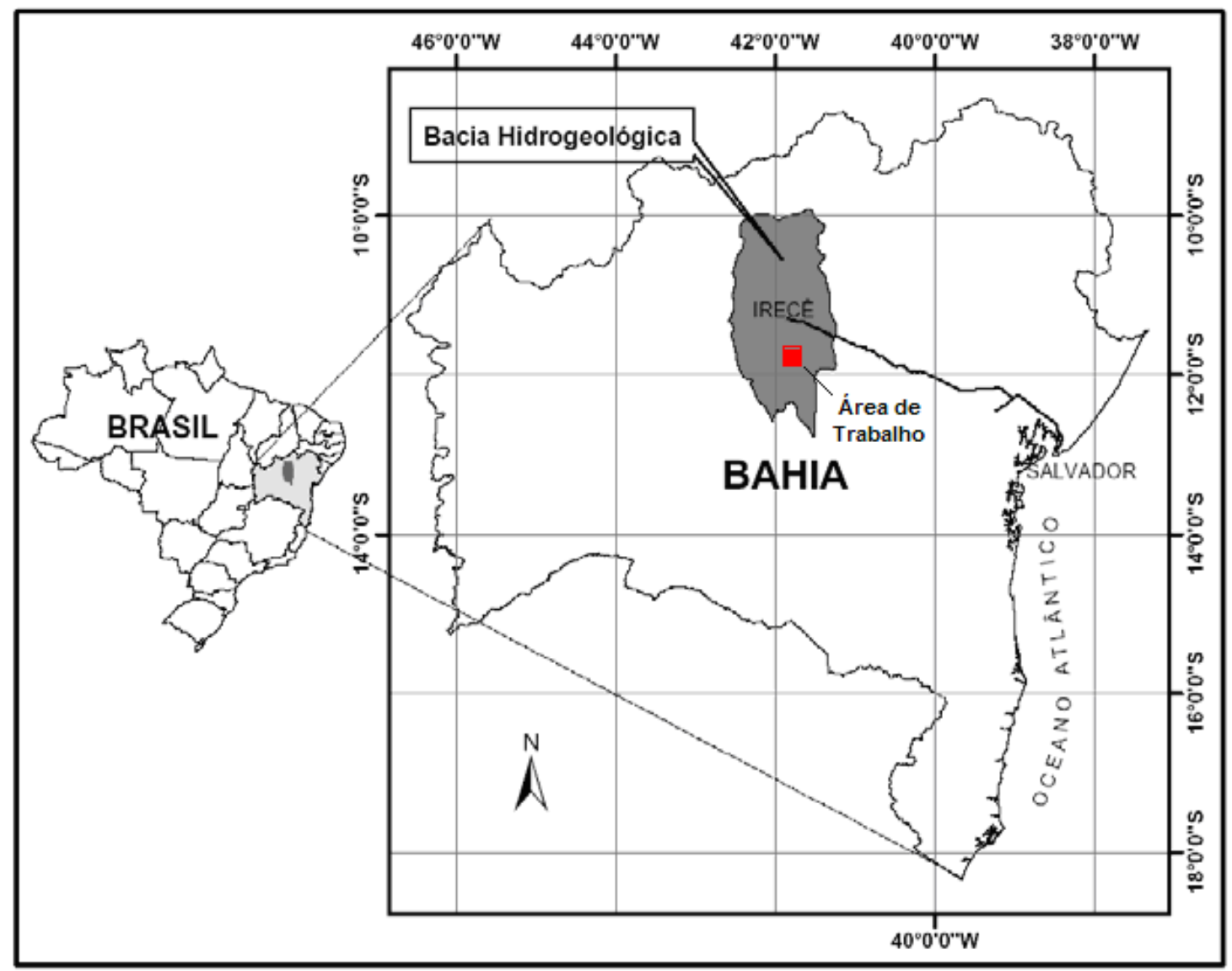

Figura 1 - Mapa de localização da área

Figure 1 - Location map of the area

$\mathrm{Na}$ área aflora fundamentalmente a Formação Salitre, que é constituída de acordo com Misi (1979) e Sampaio et al., (2001), por espessas sequências de calcarenitos, calcilutitos, calcissiltitos e dolomitos de idade Neoproterozóica, intercalados, por vezes, com sequências terrígenas su- 
bordinadas constituídas por silexitos, arenitos, siltitos, laminitos e margas, com uma espessura máxima para este pacote da ordem de $530 \mathrm{~m}$. De acordo com Pedreira et al., (1987), a área é caracterizada como paleoambiente deposicional continental e marinho de águas rasas.

Feições cársticas bem desenvolvidas caracterizam a área, que juntamente com zonas de fraturamento, constituem os reservatórios de grande expressividade do ponto de vista de recepção, armazenamento e circulação das águas subterrâneas.

\section{Isótopos estáveis e hidrogeologia}

O que permite o trabalho com isótopos estáveis é a diferença de massa e o processo de fracionamento que possibilita identificar os diferentes isótopos de um mesmo elemento. Durante mudanças de estado e reações químicas, os elementos são fracionados, o que significa dizer que ocorre uma divisão de isótopos entre duas substâncias ou fases de uma mesma substância que fornecerão diferentes razões isotópicas. $\mathrm{O}$ fracionamento isotópico, de acordo com Faure e Mensing (2005), é controlado, principalmente, pela diferença de massas dos isótopos e pela temperatura. De acordo com Kendall e Doctor (2003), o fracionamento é a mudança das proporções relativas de vários isótopos estáveis devido a reações físicas, químicas e biológicas.

A composição isotópica das águas subterrâneas para Gat (1971) é semelhante à da precipitação nas áreas de recarga. A variação de toda água meteórica é fortemente atenuada durante a sua infiltração e acumulação no solo. Essa atenuação varia com a profundidade, a superfície e a característica do embasamento geológico, mas em geral, de acordo com (Hoefs, 1997), águas subterrâneas profundas não demonstram variação sazonal nos valores de $\delta \mathrm{D}$ e $\delta^{18} \mathrm{O}$ e apresentam composição isotópica próxima à média anual de precipitações.

A composição isotópica das águas de regiões cársticas está relacionada com as fontes de carbono existentes neste ambiente, como o carbonato presente na constituição das rochas cársticas, $\mathrm{CO}_{2}$ do ar atmosférico, $\mathrm{CO}_{2}$ do ar do solo e a matéria orgânica. Processos de infiltração de água das chuvas dissolvem o $\mathrm{CO}_{2}$, presente no ar do solo, que é uma das principais fontes de carbono das águas subterrâneas, dissociando-o em $\mathrm{CO}_{3}{ }^{2-} \mathrm{e}$ $\mathrm{HCO}_{3}$. O carbono também pode ser liberado no solo pela decomposição da matéria orgânica ou através de processos de respiração das raízes das plantas (CLARK e FRITZ, 1997).

\section{MATERIAIS E MÉTODOS}

Coleta de água subterrânea para análise isotópica de oxigênio-18, deutério e carbono inorgânico dissolvido $\left(\delta^{18} \mathrm{O}, \delta \mathrm{D}\right.$ e $\left.\delta^{13} \mathrm{C}_{\mathrm{CID}}\right)$

As metodologias utilizadas para a coleta e estocagem das amostras foram determinadas de acordo com as instruções da Agência Internacional de Energia Atômica (IAEA, 2002). Os principais problemas que devem ser evitados são o fracionamento através de evaporação ou difusão do vapor de água e/ou troca isotópica com o ambiente ao redor e com o frasco de armazenamento, para tanto as amostras foram coletadas em frascos de vidro de cor âmbar de $100 \mathrm{ml}$ com tampão, para evitar a formação de bolhas e acondicionadas de forma a evitar oscilação térmica.

A coleta de amostras de água foi realizada em 36 poços tubulares da área, em duas campanhas de campo nos períodos de 2009 e 2010.

As análises das amostras coletadas foram realizadas no Laboratório de Física Nuclear Aplicada da Universidade Federal da Bahia (LFNACPGG/IF-UFBA), de acordo com a aplicação das seguintes metodologias: Razão deutériohidrogênio D/H (BRAND et al., 2000); Razão $\mathrm{O}^{18} / \mathrm{O}^{16}$ (EPSTEIN e MAYEDA, 1953); Razão $\mathrm{C}^{13} / \mathrm{C}^{12}$ do carbono inorgânico dissolvido-DIC (CRAIG, 1957).

Para a realização das análises isotópicas, foi utilizado um sistema de espectrometria de massas contendo um espectrômetro de massas de razão isotópica-MS Finnigan MAT Delta Plus e um reator automático H-Device Thermo Quest Finnigan, específico para a análise da razão D/H e sistema de preparação automático de amostras online, Gas Bench-II da Finnigan, específico para as análises do $\delta^{18} \mathrm{O}$ e $\delta^{13} \mathrm{C}_{\mathrm{CID}}$.

A análise isotópica de carbono inorgânico dissolvido (DIC) foi efetuada utilizando-se os padrões secundários Bahia: $\mathrm{PB}_{1}$ (água do mar de Salvador) e $\mathrm{PB}_{2}$ (água de cacimba da região de Irecê) para o $\delta^{18} \mathrm{O}$ e para o $\delta \mathrm{D}$ e soluções prepara-

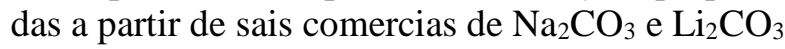
para o $\delta^{13} \mathrm{C}_{\mathrm{CID}}$.

\section{DISCUSSÃO}

\section{Análise isotópica}

A predominância de valores isotópicos negativos para as águas da Formação Salitre é sugestiva de processo de progressiva depleção dos isótopos pesados pelo efeito continental, conforme citado por Gat (1971), sugerindo que a origem dessas águas pode ser explicada pela evaporação 
das águas superficiais do Oceano Atlântico Subtropical, cujos vapores são carregados pelos ventos predominantes de Leste para Oeste. Na medida em que adentra pelo continente, o vapor sobe para maiores altitudes quando transpõe serras com picos de até $1200 \mathrm{~m}$, como a serra da Babilônia, localizada $50 \mathrm{~km}$ a Oeste de Irecê.

$\mathrm{Na}$ bacia do rio Salitre foi constatado por Santos (2008), que o $\delta^{18} \mathrm{O}$ variou no aquífero cárstico de $(-5,0 \%$ a $-1,6 \%$ ), variações muito similares às variações da área de estudo, que compreende um polígono de $250 \mathrm{~km}^{2}$. Essa situação pode ser justificada ao se analisar as médias anuais das composições isotópicas de $\delta^{18} \mathrm{O}$ para as precipitações na região obtidas na estação mais próxima, a estação de Lençóis, localizada a 52km da área, que registra precipitações bem distribuídas no intervalo de $(-0,9 \%$ a $-7,4 \%$ ), como constatado por Azevedo et al., (1991).

$\mathrm{Na}$ região de Irecê foram obtidos valores médios nas análises de isótopos de oxigênio $\left(\delta^{18} \mathrm{O}\right)$ por Siqueira $(1978)$ de $(-5,4 \%)$, Bastos
Leal e Silva (2004) de $(-4,9 \%)$ para a Bacia de Irecê, Beraldo (2005) de (-4,9\%o) e Santos (2008) de $(-4,0 \%)$ para a Bacia do rio Salitre. Esses valores são considerados muito próximos entre si.

\section{Resultados dos valores isotópicos de oxigênio- 18, deutério, e carbono inorgânico dissolvido $\left(\delta^{18} \mathrm{O}, \delta \mathrm{D}, \delta^{13} \mathrm{C}_{\mathrm{CID}}\right)$}

A análise da Figura 2 permite verificar que, no período de coleta de 2009 o coeficiente angular da curva obtida em relação à LMG (Linha Meteórica Global) foi de 6,3, enquanto que no período de coleta de 2010, o coeficiente angular foi de 6,6. Os valores obtidos indicam que as águas sofreram evaporação durante o processo de infiltração para os dois períodos de coleta, com maior efeito de fracionamento cinético no período seco. A similaridade dos resultados obtidos nas duas campanhas pode ter ocorrido em função da pequena variação dos índices pluviométricos nos períodos de amostragem considerados por NOSSA et al., (2011).

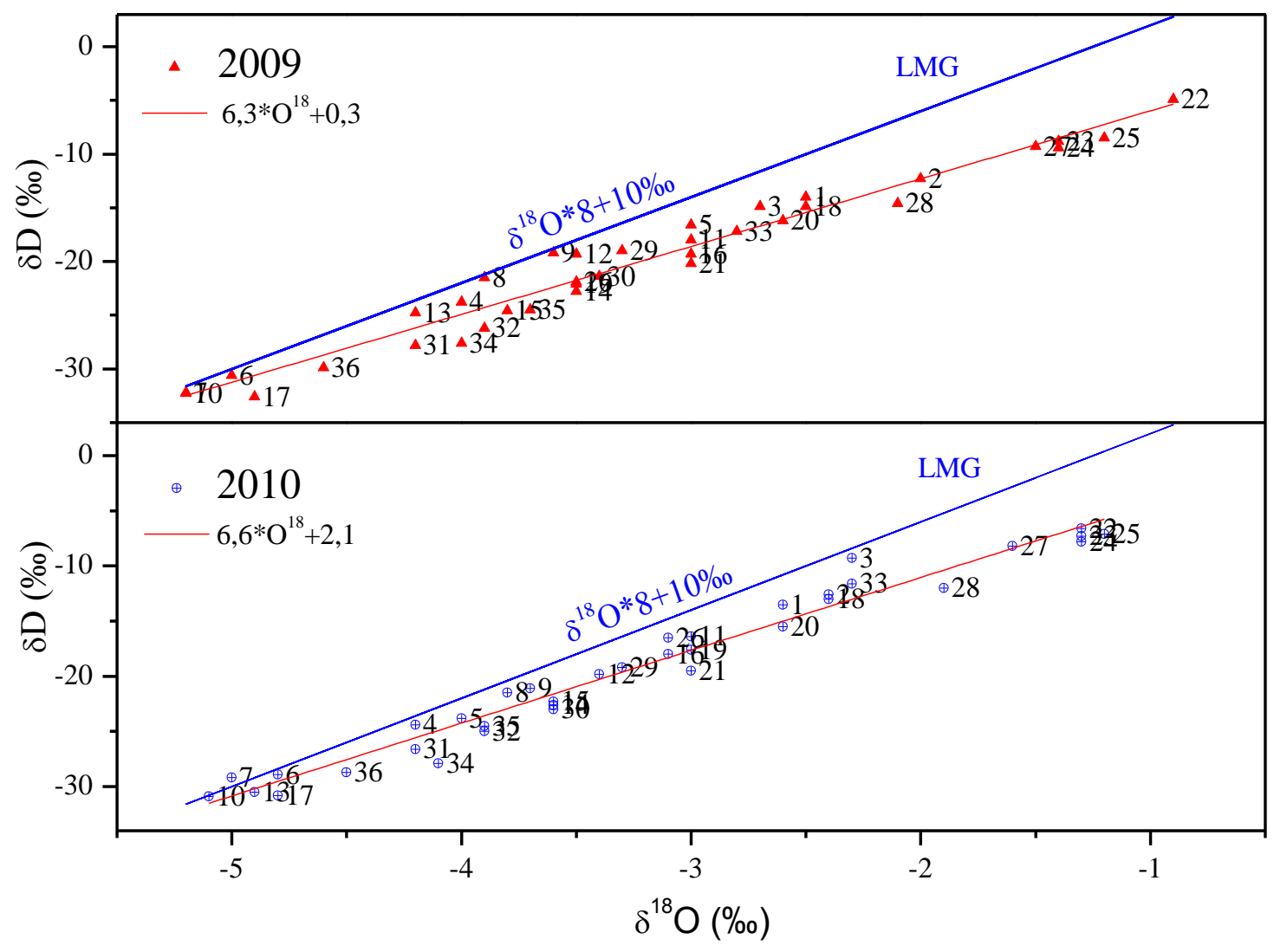

Figura 2 - Gráfico $\left(\delta^{18} \mathrm{O} \times \delta \mathrm{D}\right)$ das amostras de água de precipitação coletadas em várias partes do mundo-LMG (Linha Meteórica Global), (Rozanski et al., 1993). Aliado à representação das curvas obtidas na área para as campanhas de 2009 e 2010

Figure 2 - Graph $\left(\delta^{18} \mathrm{O} \times \delta \mathrm{D}\right)$ of water samples of precipitation collected in various parts of the world-LMG (Global Meteoric Line), (Rozanski et al, 1993). Allied to the representation of the curves obtained in the area for the 2009 and 2010 campaigns 
As análises isotópicas obtidas da coleta de águas subterrâneas em poços tubulares localizados no setor leste à montante do fluxo apresentam um menor enriquecimento isotópico do que aquelas obtidas de poços localizados no setor norte- noroeste à jusante do fluxo, demonstrando que o enriquecimento isotópico ocorre preferencialmente de Leste para Oeste, concordante com as direções preferenciais de fluxo obtidas na área (Figuras 3,4 e 5 ).

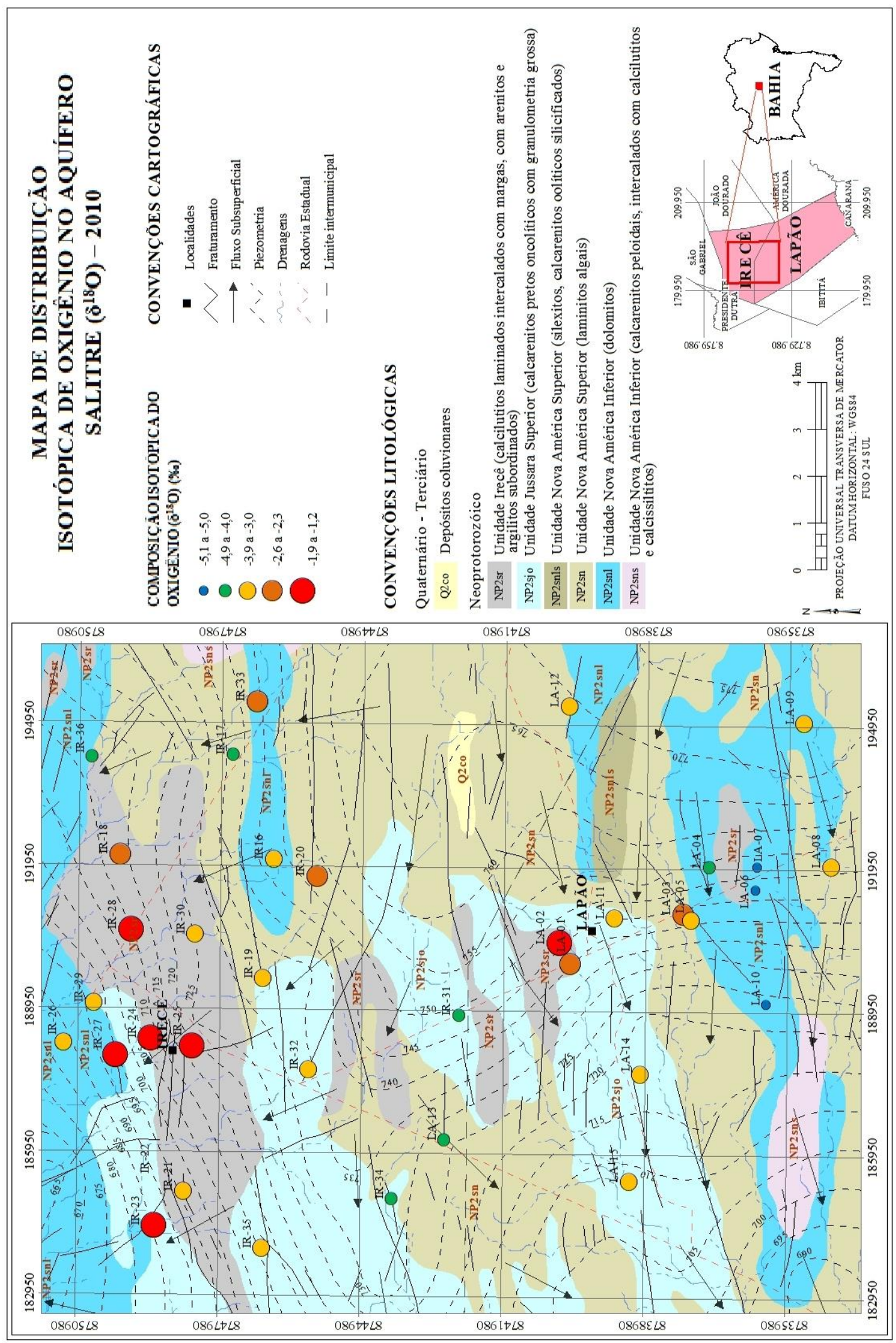

Figura 3 - Mapa de Distribuição Isotópica de Oxigênio-18 $\left(\delta^{18} \mathrm{O}\right)$ no Aquífero Salitre Figure 3 - Map of Isotopic Distribution of Oxygen-18 $\left(\delta^{18} \mathrm{O}\right)$ in Salitre Aquifer 


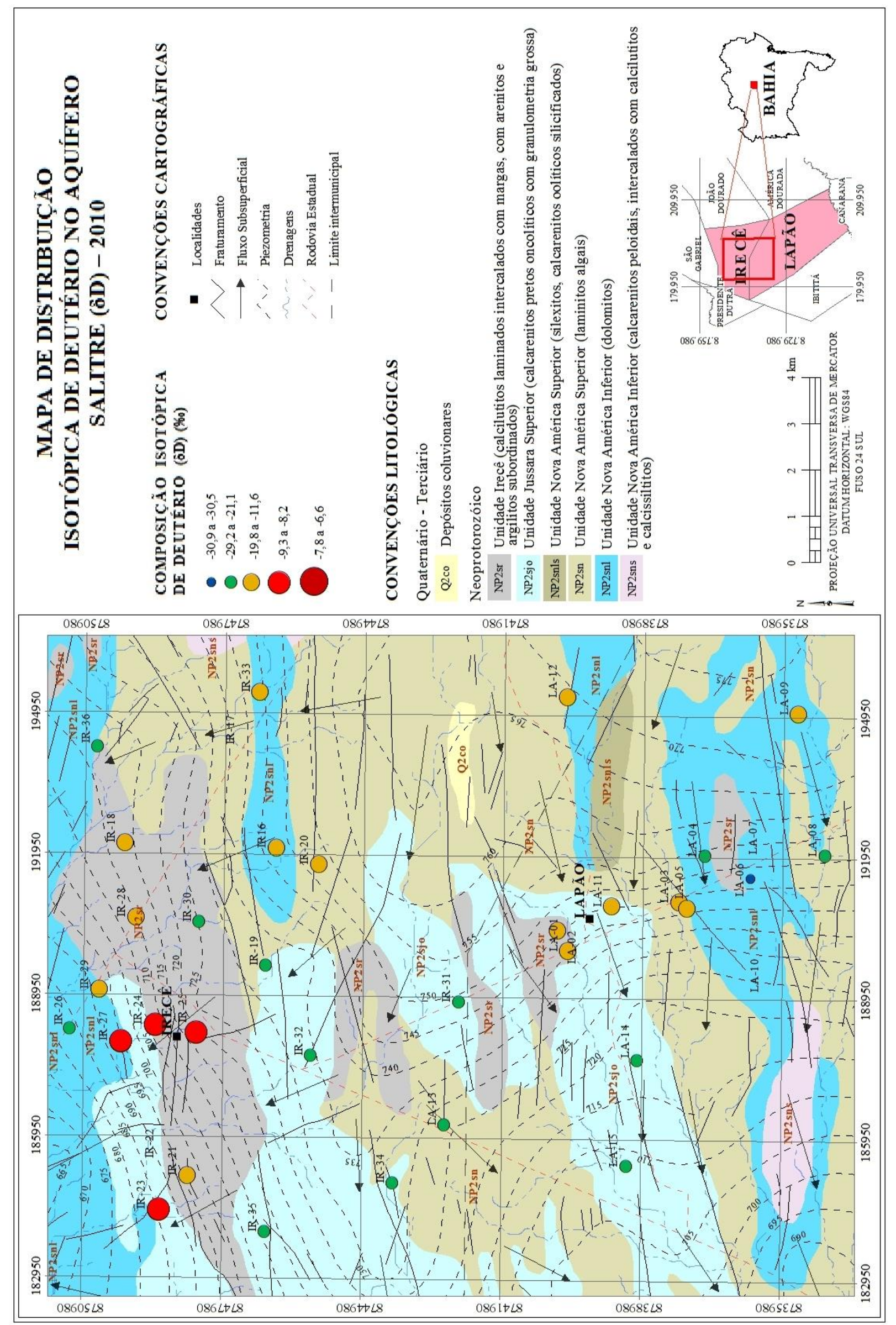

Figura 4 - Mapa de Distribuição Isotópica de Deutério $(\delta D)$ no Aquífero Salitre

Figure 4 - Map of Isotopic Distribution of Deuterium $(\delta D)$ in Salitre Aquifer 


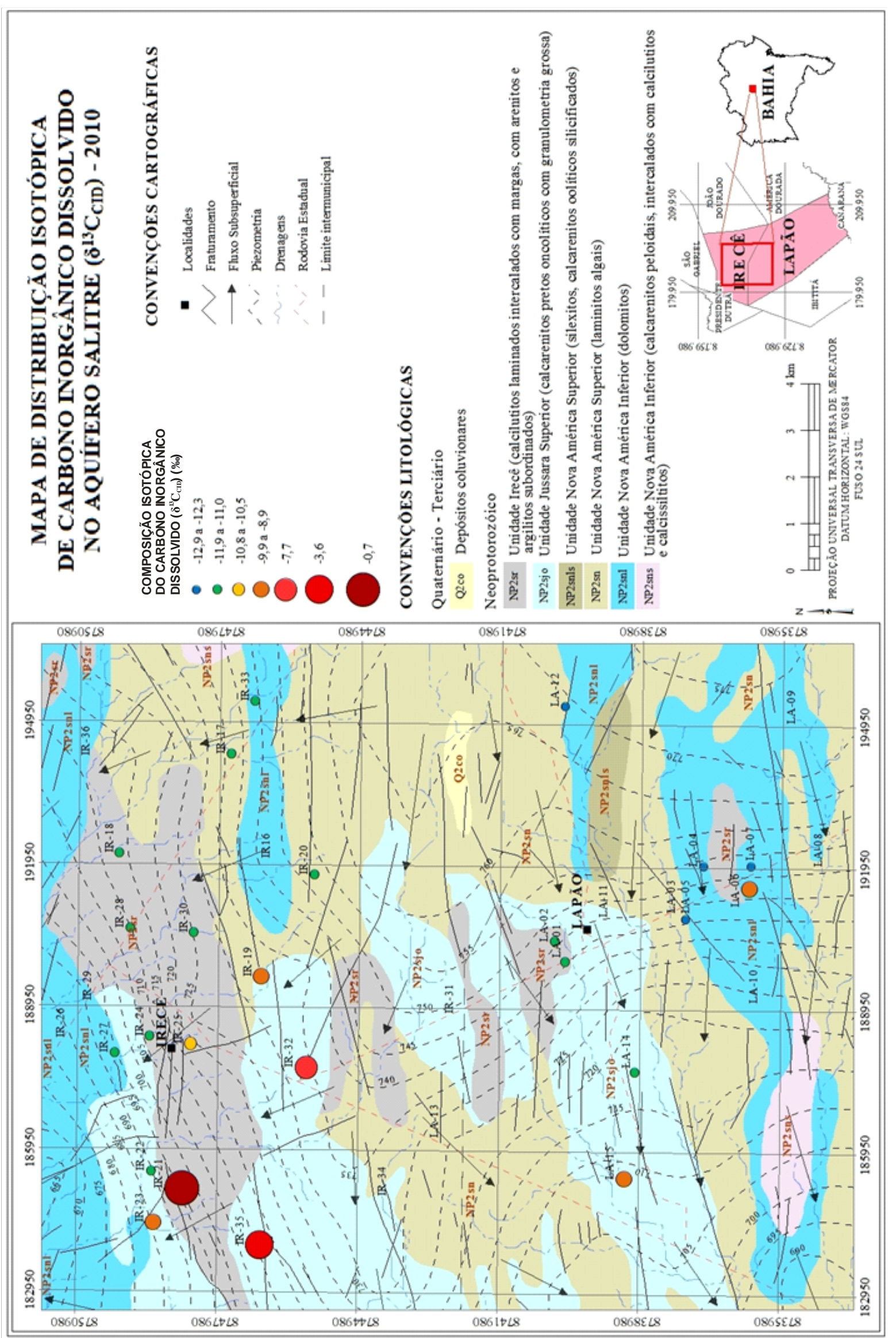

Figura 5 - Mapa de Distribuição Isotópica de Carbono Inorgânico Dissolvido $\left(\delta^{13} C_{C I D}\right)$ no Aquífero Salitre Figure 5 - Map of Isotopic Distribution of Dissolved Inorganic Carbon $\left(\delta^{13} C_{C I D}\right)$ in Salitre Aquifer 
Os valores isotópicos de oxigênio-18 $\left(\delta^{18} \mathrm{O}\right)$ variam de $(-5,2 \%$ a $-0,9 \%$ ), com média de $(-3,2 \%)$ na campanha de 2009 e de $(-5,1 \%$ a $-1,2 \%)$, com média de $(-3,2 \%)$ na campanha de 2010. Pode-se verificar que as médias são as mesmas, não havendo, portanto variação significativa dos valores analisados.

Os valores isotópicos de deutério $(\delta \mathrm{D})$ variam de $(-32,6 \%$ a $-4,9 \%)$, com média de $(-19,8 \%$ o na campanha de 2009 e de $(-30,9 \%$ a $-6,6 \%$ ), com média de $(-19,3 \%$ ) na campanha de 2010.

Os valores isotópicos de carbono inorgânico dissolvido $\left(\delta^{13} \mathrm{C}_{\mathrm{CID}}\right)$ variam de $(-14,1 \%$ a $-1,9 \%$ ), com média de (-11,5\%o) na campanha de 2009 e de $(-12,9 \%$ a $-0,7 \%)$, com média de $(-10,6 \%$ ) na campanha de 2010 .

As águas localizadas no setor nortenoroeste apresentam valores isotópicos mais elevados de oxigênio e deutério $\left(\delta^{18} \mathrm{O}\right.$ e $\left.\delta \mathrm{D}\right)$, (Figuras 3,4 e 5), o que demonstra que são águas mais evaporadas, com interações maiores com as rochas calcárias da Formação Salitre, evidenciadas pelos valores crescentes de $\delta^{13} \mathrm{C}_{\mathrm{CID}}$, concordantes com o sentido do fluxo na área.

Os intervalos de $\delta^{18} \mathrm{O}$ variam nas águas subterrâneas dos poços analisados de $(-5,2 \%$ a $-0,9 \%$ ) em 2009 e de $(-5,1 \%$ a $-1,2 \%$ ) em 2010 , com valores isotópicos médios de $\delta^{18} \mathrm{O}$ de $(-3,1 \%)$, que são similares aos valores isotópicos médios de $\delta^{18} \mathrm{O}$ obtidos de dados de precipitação na região de $(-3,2 \%)$.

Todos os valores obtidos para análise de $\left(\delta^{18} \mathrm{O}, \delta \mathrm{D}, \delta^{13} \mathrm{C}_{\mathrm{CID}}\right.$ ) são negativos; (Figuras $3,4 \mathrm{e}$ 5), o que demonstra que são águas empobrecidas em relação aos padrões VSMOW e PDB.

As amostras IR-21 e IR-35 representadas nas Figuras 3, 4 e 5, apresentam um maior enriquecimento isotópico do que as demais amostras, com valores de $\delta^{13} \mathrm{C}_{\mathrm{CID}}$ variáveis nas duas campanhas, sendo para IR-21 entre $(-4,0 \%$ e e $-3,6 \%$ ) e para IR-35 entre (-1,9\%o e -0,7\%o), respectivamente. $\mathrm{O}$ enriquecimento isotópico de $\delta^{13} \mathrm{C}_{\mathrm{CID}}$ nas águas subterrâneas nesses pontos revela uma maior interação da água com a rocha calcária nesses locais.

A distribuição do $\mathrm{pH}$ e do $\delta^{13} \mathrm{C}_{\mathrm{CID}}$, demonstra que valores de $\delta^{13} \mathrm{C}_{\mathrm{CID}}$ entre (-14,1\%o e -9,2\%o), obtidos na campanha de 2009 e entre $(-12,9 \%$ e $-7,7 \%)$, obtidos na campanha de 2010 indicam que nessas águas ocorre dominância da ação do intemperismo de carbonatos por ácido carbônico. Valores enriquecidos de $\delta^{13} \mathrm{C}_{\mathrm{CID}}$ observados nas amostras IR-21 entre $(-4,0 \%$ e $-3,6 \%$ ) e IR-35 entre $(-1,9 \%$ e $-0,7 \%$ ), revelam a dominância da ação de intemperismo de carbonatos por ácido sulfúrico, que podem estar relacionados pontualmente a concentrações de eventuais bolsões de sulfetos como pirita e calcopirita disseminados no calcário, nas proximidades desses locais.

Os valores de $\mathrm{pH}$ variaram em um intervalo de $(6,93$ a 8,27$)$ na campanha de 2009 e de $(7,41$ a 8,38$)$ na campanha de 2010 , demonstrando valores de $\mathrm{pH}$ um pouco mais elevados na campanha de 2010, referente ao período de coleta dos meses de junho e julho, com baixos índices pluviométricos. Por outro lado, os valores de $\mathrm{pH}$ mais baixos referentes à campanha de 2009 , relacionado ao período de coleta dos meses de novembro e dezembro, período chuvoso, podem refletir uma maior dissolução das rochas calcárias em contato com as águas subterrâneas do aquífero nesse período.

As análises isotópicas obtidas da coleta de águas subterrâneas em poços tubulares localizados no setor leste à montante do fluxo apresentam um menor enriquecimento isotópico do que aquelas obtidas em poços localizados no setor nortenoroeste à jusante do fluxo, demonstrando que o enriquecimento isotópico ocorre, sobretudo, de Leste para Oeste, concordante com as direções preferenciais de fluxo obtidas na área.

Os gráficos de isótopos de oxigênio $\mathrm{x}$ cloreto $\left(\delta^{18} \mathrm{O} \mathrm{Cl}^{-}\right)$e de isótopos de oxigênio $\mathrm{x}$ sólidos totais dissolvidos $\left(\delta^{18} \mathrm{O}\right.$ x STD) mostrados na Figura 6 (a) e (b) não apresentam uma relação de proporcionalidade com as águas subterrâneas analisadas, portanto, não há correlação entre o teor de cloreto na água e sua característica isotópica $\left(\delta^{18} \mathrm{O}\right)$ e $(\delta \mathrm{D})$, que são provenientes da água da chuva. Assim, esse comportamento sugere que a origem da salinidade dessas águas deve estar relacionada com a sua interação com a sequência carbonática, bem como, com o processo de evapotranspiração, como constatado por NOSSA (2011).

Em uma Análise de Componentes Principais (PCA), define-se a estrutura dos dados através de gráficos de scores e loadings cujos eixos são componentes principais (PCs) nos quais os dados são projetados. Os scores fornecem a projeção dos dados no espaço das PCs, enquanto que os loadings fornecem a significância relativa em relação às variáveis originais. Como as PCs são ortogonais, é possível examinar as relações entre amostras e variáveis através dos gráficos dos $\mathrm{sco}$ res e dos loadings. O estudo do conjunto de scores e loadings ainda permite estimar a influência de cada variável em cada amostra. 
Para entender as correlações entre os isótopos ambientais e as variáveis físico-químicas, utilizamos a técnica estatística de Análise de Componentes Principais (PCA). A visualização espacial das amostras de água coletadas em cada ponto tornou possível a análise e identificação da relação entre as características dos dados e seus possíveis agrupamentos.

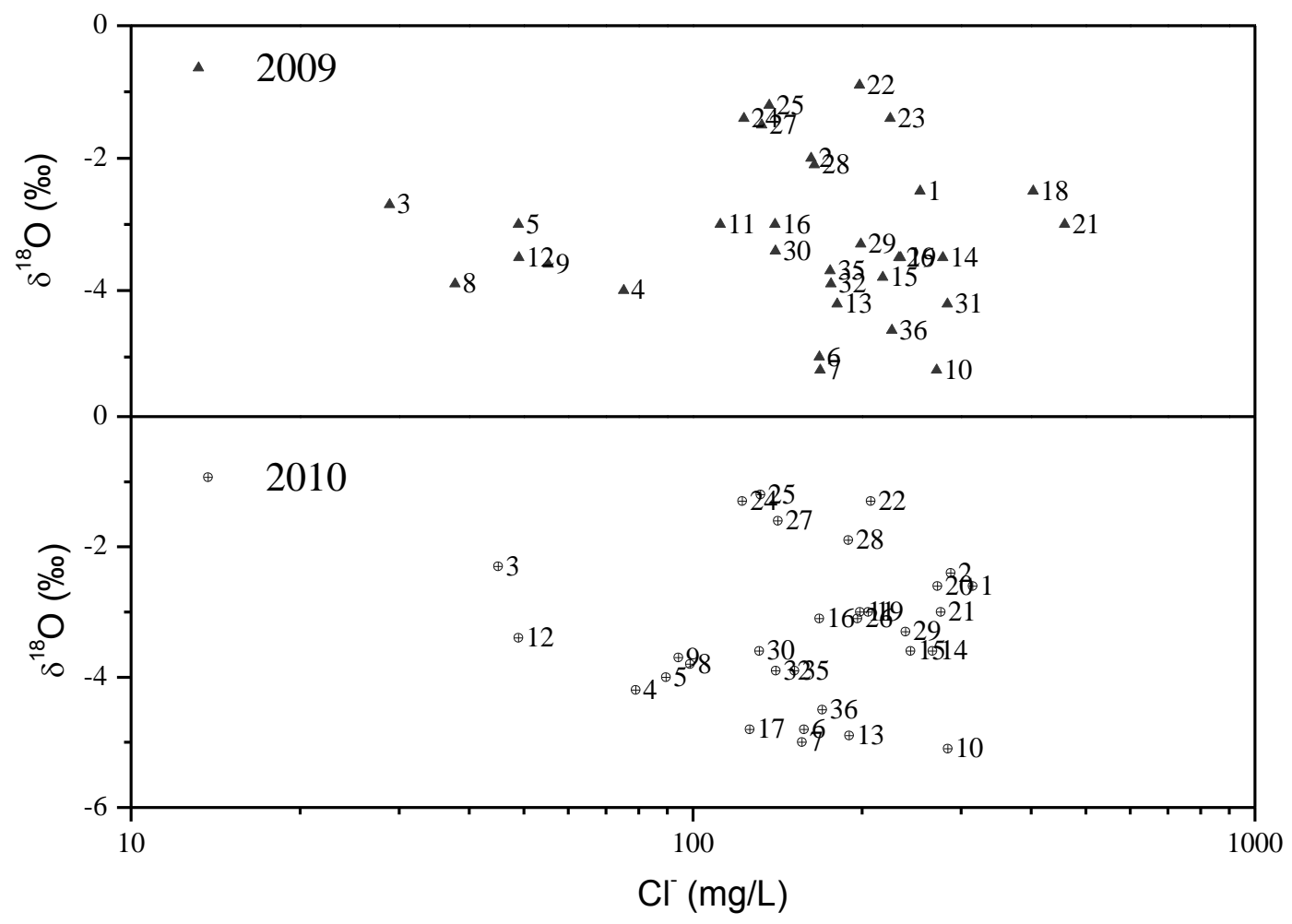

(a)

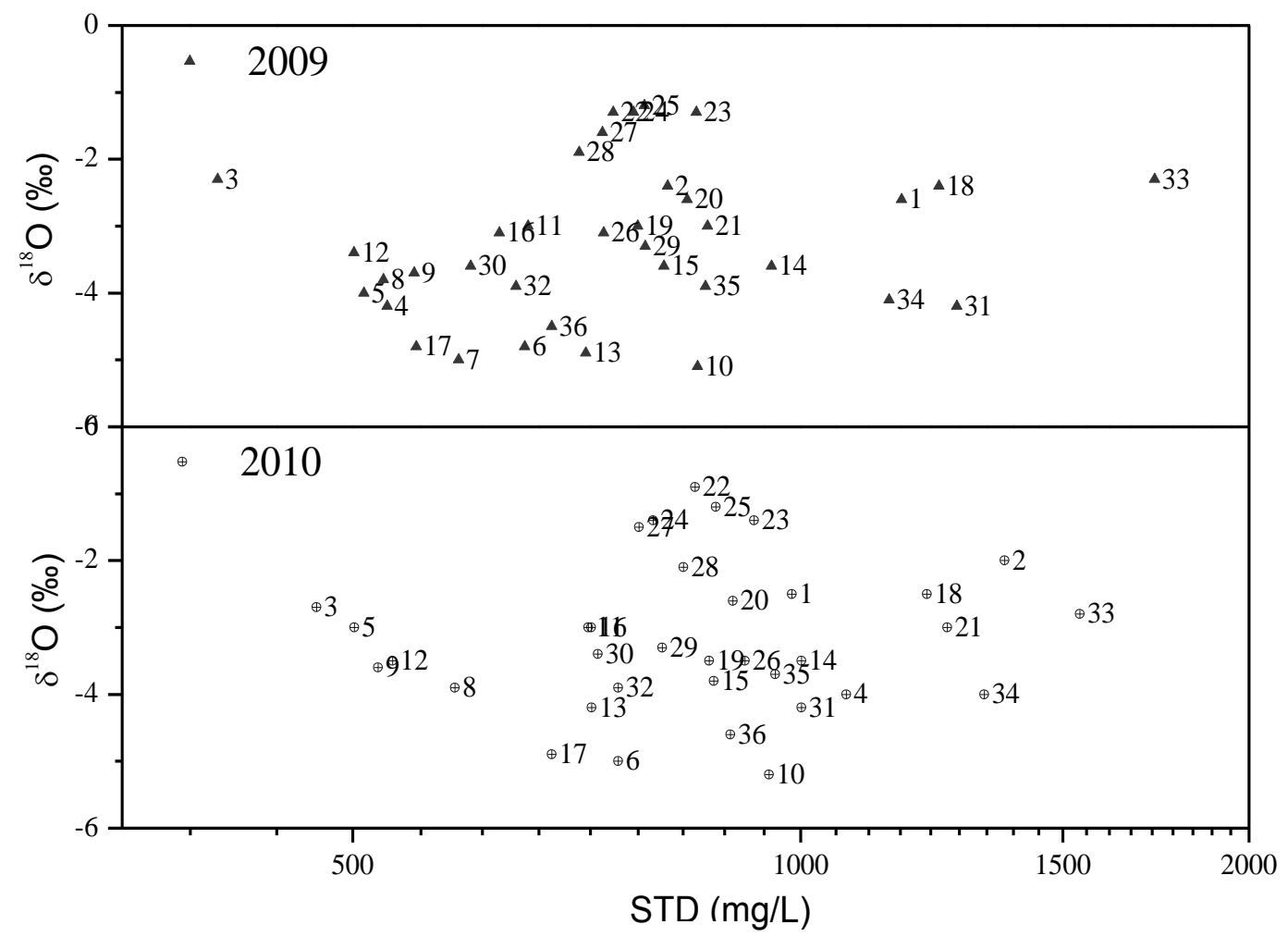

(b)

Figura 6 - Representação gráfica das variações de (a) $\mathrm{Cl}^{-} \times \delta^{18} \mathrm{O}$ e (b) $\mathrm{STD} \times \delta^{18} \mathrm{O}$ referente às campanhas de 2009 e 2010

Figure 6 - Graphical representation of the variations of (a) $\mathrm{Cl}^{-} \times \delta^{18} \mathrm{O}$ and (b) STD $\mathrm{S} \delta^{18} \mathrm{O}$ referring for the 2009 and 2010 campaigns 
A Figura 7 (a) e (b) ilustra o PC1 x PC2 de loadings para as variáveis isotópicas e hidroquímicas de 2009 e 2010, onde não se observa correlação dos valores isotópicos de oxigênio-18 $\left(\delta^{18} \mathrm{O}\right)$ e deutério $(\delta \mathrm{D})$ com sólidos totais dissolvidos (STD), ou seja, demonstram que além da evaporação, existem outros processos que contribuem com a salinização das águas subterrâneas, como por exemplo, a transpiração realizada pelas plantas.

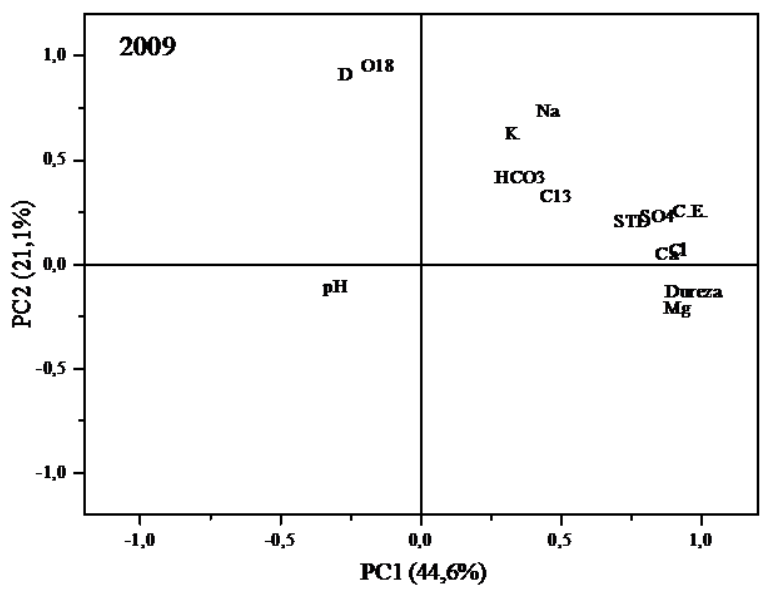

(a)

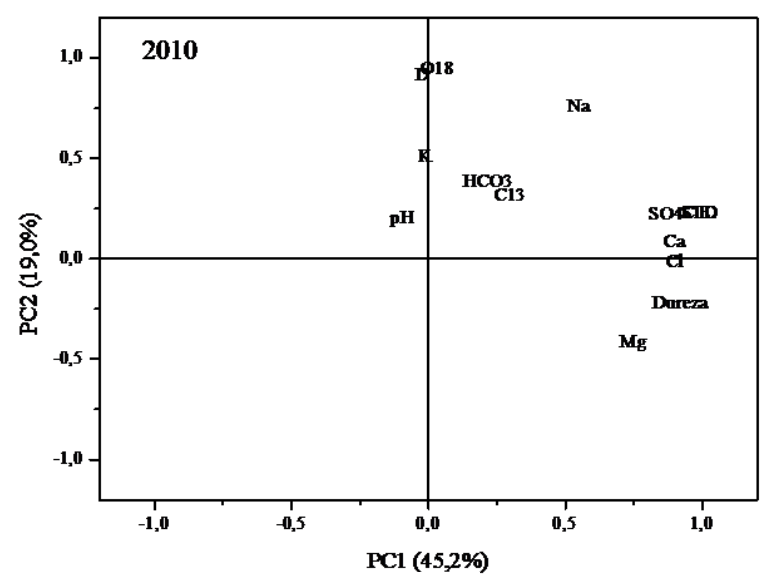

(b)

Figura 7 - Gráfico de loadings para as variáveis isotópicas e hidroquímicas. Sendo (a) 2009 e (b) 2010

Figure 7 - Plot of loadings for isotopic and hydrochemical variables. Being (a) 2009 and (b) 2010

Na Figura 7 (a) e (b), pode-se observar, de acordo com a Análise de Componentes Principais (PCA), que o carbono inorgânico dissolvido $\left(\delta^{13} \mathrm{C}_{\mathrm{CID}}\right)$ não apresenta relação direta com os parâmetros cálcio, magnésio, bicarbonato e sulfato, indicando que o fracionamento isotópico estaria relacionado em maior quantidade ao efeito das precipitações na área.
A Figura 8 (a) e (b) ilustra a primeira componente principal (PC1) x (PC2) segunda componente principal, para as amostras, sendo respectivamente 2009 e 2010. Nos gráficos de PC1 x PC2 as amostras estão agrupadas pela semelhança de suas variáveis, especialmente pelo teor dos componentes principais. Através da técnica PCA verificou-se que, com as duas primeiras componentes principais é possível descrever $65,7 \%$ dos valores, sendo $44,6 \%$ da variância total descrita pela (PC1) e $21,1 \%$ pela $\mathrm{PC} 2$, para o período de 2009 e 64,2\% dos valores, sendo $45,2 \%$ da variância total descrita pela (PC1) e 19,0\% pela PC2, para o período de 2010.

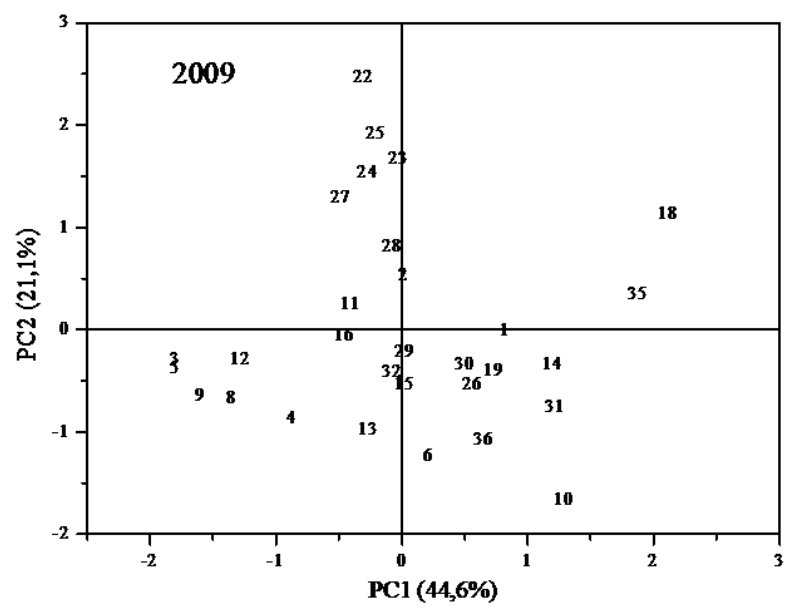

(a)

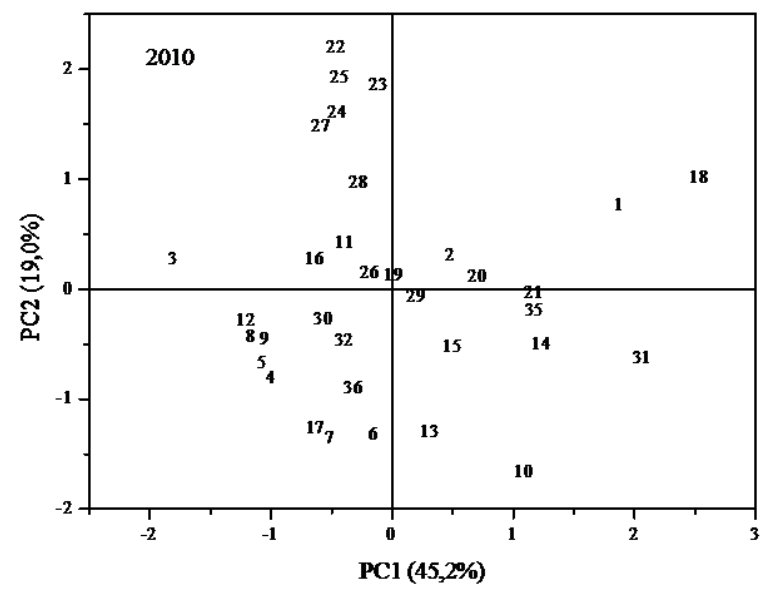

(b)

Figura 8 - Gráfico dos de PC1 x PC2 das amostras referentes às campanhas de (a) 2009 e (b) 2010

Figure 8 - Graph of PC1 x PC2 concerning the samples of campaigns (a) 2009 and (b) 2010

$\mathrm{Na}$ distribuição isotópica do carbono inorgânico dissolvido $\left(\delta^{13} \mathrm{C}_{\mathrm{CID}}\right)$ com os elementos (a) $\mathrm{Ca}^{+2} \mathrm{e}$ (b) $\mathrm{Mg}^{+2}$, pode-se verificar que as 
amostras, de maneira geral, apresentam valores elevados desses elementos, indicando que as águas que infiltram nesses aquíferos dissolvem preferencialmente minerais carbonáticos como calcita e dolomita.

A distribuição observada na Figura 8 (a) e (b) sugere que o bicarbonato resulta do intemperismo de carbonatos por $\mathrm{H}_{2} \mathrm{CO}_{3}$, indicando uma maior contribuição de processos de dissolução de carbonatos por $\mathrm{H}_{2} \mathrm{CO}_{3}$ do que por $\mathrm{H}_{2} \mathrm{SO}_{4}$. Dessa forma, os processos de dissolução de carbonatos onde predominaria o $\mathrm{H}_{2} \mathrm{SO}_{4}$, poderiam ocorrer em locais pontuais, em que houvesse um enriquecimento do $\delta^{13} \mathrm{C}_{\mathrm{CID}}$, em função da ocorrência de sulfetos na área. Os valores médios obtidos para as concentrações de $\mathrm{SO}_{4}{ }^{-2}$ na área variam de $115 \mathrm{mg} / \mathrm{L}$ a $120 \mathrm{mg} / \mathrm{L}$, com valores máximos de $306,77 \mathrm{mg} / \mathrm{L}$ na campanha de 2009 e $295,07 \mathrm{mg} / \mathrm{L}$ na campanha de 2010.

\section{CONCLUSÕES}

As análises de isótopos estáveis permitiram constatar que a origem da salinização nas águas do aquífero Salitre, pode estar tanto relacionada com a sua interação com a sequência carbonática que caracteriza a Formação Salitre, como com processos de evaporação, podendo ter contribuição também da transpiração realizada pelas plantas. Isso pode ser diagnosticado nos resultados obtidos em amostras de águas subterrâneas coletadas em poços tubulares localizados no setor leste da área, à montante do fluxo, que apresentam um menor enriquecimento isotópico do que aquelas obtidas em poços localizados no setor nortenoroeste, à jusante do fluxo, com valores isotópicos mais elevados de oxigênio-18 $\left(\delta^{18} \mathrm{O}\right)$ e deutério $(\delta \mathrm{D})$, demonstrando que são águas mais evaporadas e submetidas a interações com as rochas calcárias por um intervalo maior de tempo. O que pode ser constatado pelos valores crescentes de carbono inorgânico dissolvido $\left(\delta^{13} \mathrm{C}_{\mathrm{CID}}\right)$, que evidencia o fato de que o enriquecimento isotópico

\section{REFERÊNCIAS}

ANDREO, B.; LIÑAN, C.; CARRASCO, F.; JIMÉNEZ DE CISNEIROS, C.; CABALLERO, F.; MUDRY, J. Influence of rainfall quantity on the isotopic composition $\left({ }^{18} \mathrm{O}\right.$ and $\left.{ }^{2} \mathrm{H}\right)$ of water in mountainous areas. Application for groundwater research in the Yunquera-Nieves karst aquifers (S Spain). Applied Geochemistry, 19, 561$574,2004$.

AZEVEDO, A. E. G.; CABRAL F. C. F.; FERREIRA C. Caracterização isotópica das precipitações do estado da Bahia e de águas subterrâneas da Bacia de Tucano. 2. International Congress of the Brazilian Geo- ocorre, sobretudo, no sentido (E-W), concordante com as direções preferenciais de fluxo obtidas na área.

Valores isotópicos negativos de $\delta^{13} \mathrm{C}_{\mathrm{CID}}$ indicam uma maior contribuição de processos de dissolução de carbonatos por $\mathrm{H}_{2} \mathrm{CO}_{3}{ }^{-}$do que por $\mathrm{H}_{2} \mathrm{SO}_{4}$. Os processos de dissolução de carbonatos predominantemente por $\mathrm{H}_{2} \mathrm{SO}_{4}$ podem ocorrer com o enriquecimento de $\delta^{13} \mathrm{C}_{\mathrm{CID}}$, em função da oxidação de sulfetos, como a pirita, presente nas águas subterrâneas da área.

No período de coleta de 2009 a inclinação da curva obtida em relação à LMG (Linha Meteórica Global) na área foi de 6,3, enquanto que no período de coleta de 2010, a inclinação da curva foi de 6,6. Esses resultados indicam que as águas sofreram evaporação durante o processo de infiltração nos dois períodos de coleta, com maior efeito de fracionamento cinético no período seco. Todos os valores obtidos nas análises isotópicas de oxigênio-18, deutério e carbono inorgânico dissolvido $\left(\delta^{18} \mathrm{O}, \delta \mathrm{D}\right.$ e $\left.\delta^{13} \mathrm{C}_{\mathrm{CID}}\right)$ são negativos, o que demonstra que são águas empobrecidas em relação aos padrões VSMOW e PDB.

Analisando os dados históricos obtidos na Bacia de Irecê, foi possível constatar que nas análises de $\left(\delta^{18} \mathrm{O}, \delta \mathrm{D}\right.$ e $\left.\delta^{13} \mathrm{C}_{\mathrm{CID}}\right)$, foram diagnosticados valores médios muito próximos dos valores encontrados, corroborando o comportamento isotópico da área, que revela que não houve uma variação isotópica significativa do aquífero Salitre ao longo dos últimos 30 anos.

\section{AGRADECIMENTOS}

À CPRM - Serviço Geológico do Brasil, pelo apoio efetuado nos trabalhos de campo e nas análises químicas, realizadas pelo LAMIN - Laboratório de Análises Minerais (CPRM/RJ) e ao LFNA - Laboratório de Física Nuclear da Universidade Federal da Bahia, pela realização das análises isotópicas.

physical Society, São Paulo (SP), V. II, p.1023-1027, 1991.

BAKALOWICZ, M. La zone d'infiltration des aquiferes karstiques. Méthodes d'étude. Structure et fonctionnement, Hydrogéologie 4, 3-21, 1995.

BASTOS LEAL, L. R.; SILVA, H. P. da (Org.) Modelização da dinâmica hidrológica e instrumentos para a gestão do sistema aquífero-rio das bacias hidrográficas dos rios Verde e Jacaré. Relatório Final. Salvador: 2004. Superintendência de Recursos Hídri- 
cos do Estado da Bahia e Universidade Federal do Estado da Bahia. Projeto Concluído. Área: região semiárida do estado da Bahia.

BERALDO, V. J. Estudo isotópico $\left(\delta^{2} \mathrm{H}\right.$ e $\left.\delta^{18} \mathrm{O}\right)$ e hidroquímica na região de Irecê, Bahia: contribuição ao entendimento do ciclo hidrológico regional. Salvador, Bahia, Brasil, 2005. 84p. Dissertação (Mestrado em Geoquímica e Meio Ambiente). Universidade Federal da Bahia.

BRAND, W. A.; AVAK, H.; SEEDORF, R.; HOFMANN, D.; CONRADI, T. New methods for fully automated isotope ratio determination from hydrogen at the natural abundance level. Geoph. Prosp. (28):967-976, 2000.

CARRASCO, F.; ANDREO, B.; LIÑÁN, C.; MUDRY, J. Contribution of stable isotopes to the understanding of the unsaturated zone of a carbonate aquifer (Nerja Cave, southern Spain). C. R. Geoscience 338, 12031212, 2006

CLARK, I.; FRITZ, P. Environmental isotopes in hydrogeology. 2ed. Lewis Publishers, Boca Raton, 328p, 1997.

CRAIG, $\mathrm{H}$. Isotope standards for carbon and oxygen and conection factors for mass spectrometric analysis of carbon dioxide. Geochimica et Cosmochimica Acta. (12):133-149, 1957.

CRUZ-SAN, JULIÁN, J.; ARAGUÁS, L.; ROZANSKI, K.; BENAVENTE, J.; CARDENAL, J.; HIDALGO, M. C.; GARCÍA-LOPEZ, S.; MARTÍNEZ-GARRIDO, J. C.; MORAL, F.; OLIAS, M. Sources of precipitation over South-Eastern Spain and groundwater recharge. An isotropic study, Telus, 44b, 226-236, 1992.

EPSTEIN, S.; MAYEDA, T. Variations of ${ }^{18} \mathrm{O}$ content of waters from natural sources. Geochimica et Cosmochimica Acta. (4):213-221p, 1953.

FAURE, G.; MENSING, T. M. Isotopes: principles and applications. John Wiley e Sons. Ed., 897p, 2005.

GAT, J. R. Comments on stable isotope method in regional groundwater investigation. Water Resource Research, (7):980-993, 1971.

HOEFS, J. Stable isotope geochemistry. Ed. Springer-Verlag, Berlin, 4 ed., 201p, 1997.

IAEA. Instrumentation and control systems important to safety in nuclear power plants: safety guide. Vienna: International Atomic Energy Agency, 2002.

KENDALL, C.; DOCTOR, D. H. Stable isotope applications in hydrologic studies. In: DREVER, J. I. (ed.) Surface and ground warter, weathering, and solis. HOLLAND, H. D.; TUREKIAN, K. K. (org.) Treatise on Geochemistry, V. 5. p.319-364, 2003.

LASTENNET, $R$. Rôle de la zone non saturée dans le fonctionnement des aquifers karstiques. Approche par l'étude physic-chimíque et isotopique du signal d'entée et des exutoires du massif de Ventoux (Vaucluse). These, université d'Avignon, 1994.
MISI, A. O Grupo Bambuí no estado da Bahia. In: INDA, H. A. V. (Ed.) Geologia e recursos minerais do Estado da Bahia: Textos Básicos. Salvador: CPRM. v. 1. p.119-154, 1979.

MUDRY, J. Sur l'origine des gradients des teneurs isotopiques et géochimiques dans les eaux karstiques du Jura (France), Journal of Hydrology, 50, 167-178, 1981.

NOSSA, T. C. B. Avaliação da vulnerabilidade do aquífero cárstico Salitre - Bahia, através de análises hidroquímicas, isotópicas e aplicação da metodologia COP. Salvador, Bahia, Brasil, 2011, 226p. Tese (Doutoramento em Geologia), Universidade Federal da Bahia.

NOSSA, T. C. B.; BASTOS LEAL, L. R; ZUCCHI, M. do $R$. Hidroquímica e índices de saturação dos minerais do sistema aquífero cárstico Salitre na região de IrecêLapão, Bahia, Brasil. Anais in: II Congresso Internacional de Meio Ambiente Subterrâneo, São PauloSP. 2011.

O'LEARY, M. H. Carbon isotopes in photosynthesis. BioScience. (38):328, 1988

PEDREIRA, A. J.; ROCHA, A. J. D.; COSTA, I. V. G. da; MORAIS FILHO, J. C. Projeto Bacia de Irecê-II: relatório final. Salvador, CPRM, 1987.

ROZANSKI, K.; ARAGUÁS-ARAGUÁS, L.; GONFIANTINI, R. Isotopic patterns in modem global precipitation. In: SWART, P. K.; LOHMANN, K. C.; MCKENZIE, J.; SAVIN, S. (ed.) Climate change in continental isotopic records. Geophysical Monograph Series, 78, AGU, Washington, D.C. p. 1-36, 1993.

SAMPAIO, A. R. (Org). Programa Levantamentos Geológicos Básicos do Brasil - PLGB. Jacobina. Folha SC 24-y-C, Estado da Bahia. Escala 1:250.000. Relatório Final: Brasília. CPRM/DIEDIG/DEPAT, 2001.

SANTOS, C. P. L. dos. Análise dos processos de salinização das águas subterrâneas da bacia do rio Salitre por meio de traçadores ambientais. Salvador, Bahia, Brasil, 2008. 125p. Tese (Doutoramento em Geologia), Universidade Federal da Bahia.

SIQUEIRA, A. F. O uso dos dados isotópicos e químicos como indicadores de origem das águas e sais dissolvidos no aquífero calcário Bambuí, IrecêBahia. Salvador, Bahia, Brasil, 1978. 86p. Dissertação (Mestrado em Geoquímica), Universidade Federal da Bahia.

VALLEJOS, A.; PULIDO BOSCH, A.; MARTÍNROSALES, W..; CALVACHE, M. L. Contribution of environmental isotopes to the understanding of complex hydrologic systems. A case study: Sierra de Gador, SE Spain, Earth Surf. Processes Landforms 22 , 1157-1168, 1997.

VOGEL, J. C. Variability of carbon isotope fractionation during photosynthesis. In: J. R. EHLERINGER, A. E. Hall. Plant Carbon - Water Relations. Academic Press, San Diego. CA:29-38, 1993. 
VRBA, J.; SOBLSEK, P. Groundwater monitoring. En

Geology and Environment International Manual in three volumes. UNESCO. UNEP, 1988. 